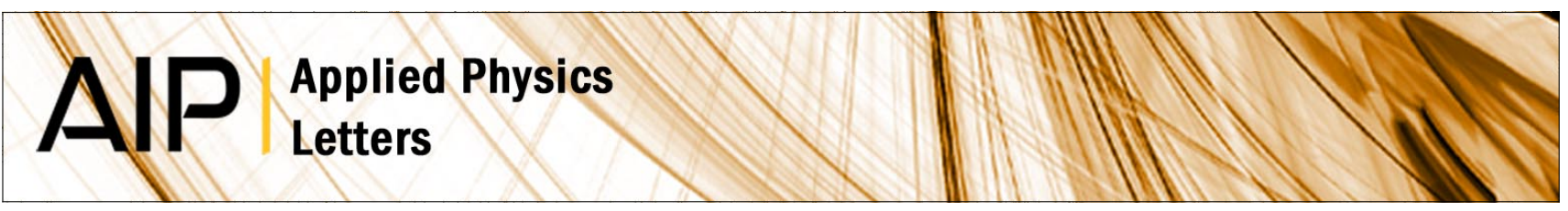

\title{
Dropping of electromagnetic waves through localized modes in three- dimensional photonic band gap structures
}

Mehmet Bayindir and E. Ozbay

Citation: Appl. Phys. Lett. 81, 4514 (2002); doi: 10.1063/1.1528733

View online: http://dx.doi.org/10.1063/1.1528733

View Table of Contents: http://apl.aip.org/resource/1/APPLAB/v81/i24

Published by the American Institute of Physics.

Additional information on Appl. Phys. Lett.

Journal Homepage: http://apl.aip.org/

Journal Information: http://apl.aip.org/about/about_the_journal

Top downloads: http://apl.aip.org/features/most_downloaded

Information for Authors: http://apl.aip.org/authors

\section{ADVERTISEMENT}

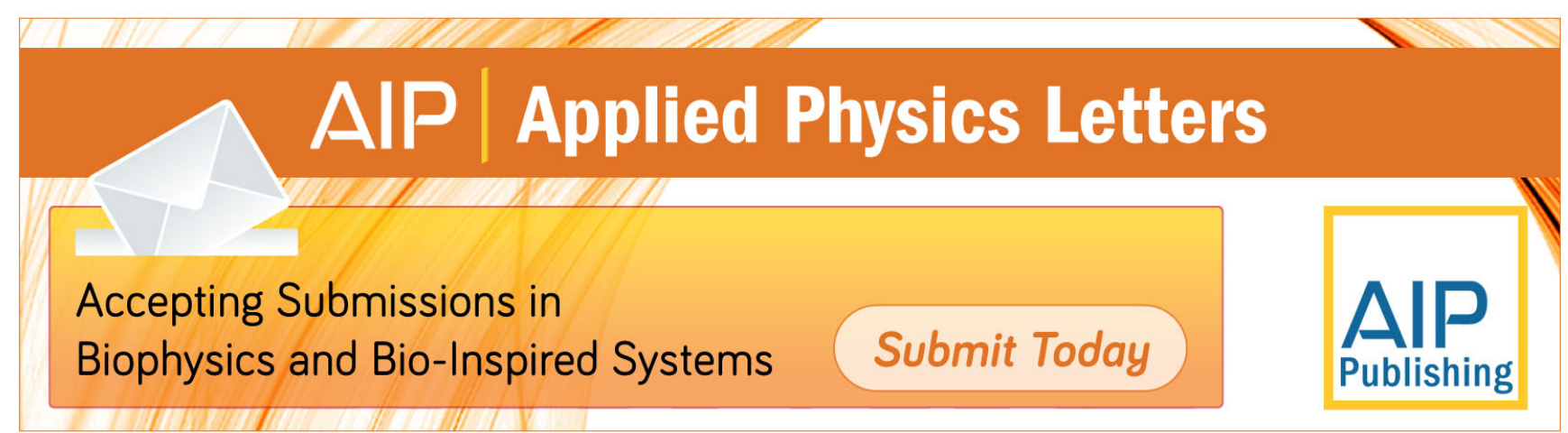




\title{
Dropping of electromagnetic waves through localized modes in three-dimensional photonic band gap structures
}

\author{
Mehmet Bayindir ${ }^{\mathrm{a})}$ and E. Ozbay \\ Department of Physics, Bilkent University, Bilkent, 06533 Ankara, Turkey
}

(Received 20 May 2002; accepted 8 October 2002)

\begin{abstract}
We experimentally demonstrate trapping and dropping of photons through localized cavity modes in three-dimensional layer-by-layer photonic crystal structures. By creating acceptor- and donor-like cavities which are coupled to a highly confined waveguide (HCW), we drop selected frequencies from the waveguide mode. Tunability of the demultiplexing structures can be achieved by changing the properties of cavities and the coupling between the cavity and the HCW. (C) 2002 American Institute of Physics. [DOI: 10.1063/1.1528733]
\end{abstract}

In recent years, there has been much interest in the possible realization of photonic crystals for the design of optical components and circuits. ${ }^{1}$ Up to now, various applications of photonic band gap (PBG) structures have been experimentally demonstrated including waveguides,${ }^{2-5}$ power splitters, ${ }^{4,6,7}$ switches, ${ }^{6}$ lasers, ${ }^{8}$ and wavelength division multiplexing (WDM).$^{9-11}$

Photonic band gap structures can also be used to construct the optical add-drop filters which can be used effectively in WDM applications. The first photonic crystal based WDM structure was proposed by Fan et al. using resonant tunneling phenomena between two waveguides via cavities. ${ }^{9}$ Kosaka et al. reported WDM filters using superprism phenomena. ${ }^{10}$ Noda and his co-workers proposed and experimentally demonstrated trapping and dropping of photons via cavity-waveguide coupling in two-dimensional (2D) photonic crystal slabs. ${ }^{11}$ Nelson et al. reported the wavelength separation using one-dimensional (1D) dielectric multilayer stacks. ${ }^{12}$ Recently, various types of WDM structures in 2D photonic crystals have also been reported. ${ }^{13-16}$

Most of the foregoing applications are built around 2D PBG structures. However, to avoid the leakage problem in 2D structures, either one has to extend the size of the photonic crystal in the vertical direction, or use a strong indexguiding mechanism in the vertical direction. ${ }^{17}$ Another way to eliminate the leakage problem is to use 3D photonic crystals in such applications. ${ }^{18,19}$ Even if the fabrication of 3D photonic crystals is not easy, the introduction of 3D photonic structures is twofold. First, 3D crystals have high rejection rates. Second, one can confine light within a very small volume of 3D structures, and this property leads to very high quality factors. ${ }^{20}$

Recently, a method for full confinement of the electromagnetic (EM) waves utilizing 3D layer-by-layer photonic crystals was reported. ${ }^{4,19,21,22}$ A single rod is removed from an otherwise periodic crystal in order to construct waveguides, which are also called highly confined waveguides (HCWs). Photons can propagate through the vacancy of the missing rod [see Fig. 1(a)] without any radiation

a) Author to whom correspondence should be addressed; present address: Research Laboratory of Electronics, Massachusetts Institute of Technology, Cambridge, MA 02139; electronic mail: mehmet@mit.edu losses for certain wavelengths. Various photonic components such as waveguides, waveguide bends, and power dividers, which are built around HCWs, have been experimentally demonstrated. ${ }^{4}$ In this letter, we demonstrate a method for dropping photons in 3D layer-by-layer photonic crystals.

A layer-by-layer dielectric based photonic crystal ${ }^{20,23,24}$ is used to construct the demultiplexing structures. The crystal consists of square shaped alumina rods having a refractive index of 3.1 at microwave frequencies and dimensions of $0.32 \mathrm{~cm} \times 0.32 \mathrm{~cm} \times 15.25 \mathrm{~cm}$. A center-to-center separation between the rods of $1.12 \mathrm{~cm}$ is chosen to yield a dielectric filling ratio of $\sim 0.26$. The unit cell consists of four layers that have the symmetry of a face-centered-tetragonal (fct) crystal structure. The crystal exhibits a three-dimensional

(a)

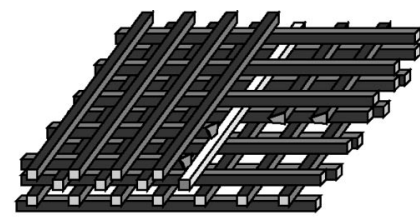

(b)

(c)
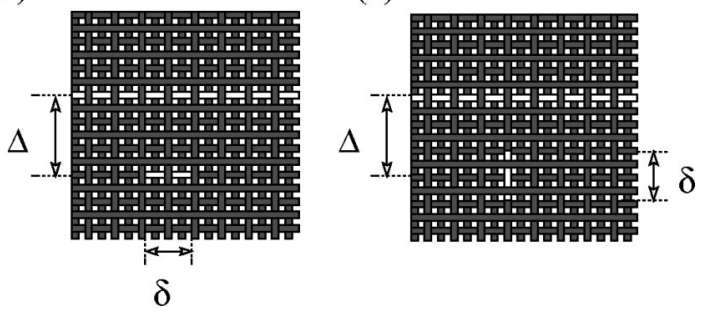

(d)

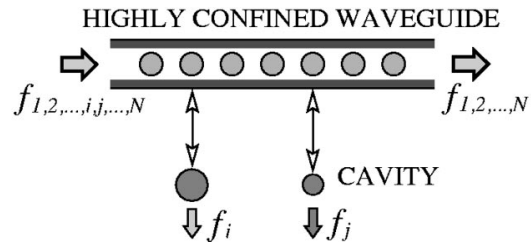

FIG. 1. Dropping of electromagnetic waves in 3D photonic crystal structures. (a) Schematics of the highly confined waveguides. The EM waves are tightly confined, and propagate along the vacancy of a single rod (white rod) removed from a 3D layer-by-layer photonic crystal. (b), (c) Proposed configurations for demultiplexing applications in photonic crystals. Some portion, $\delta$, of a single rod is removed to construct an acceptor-like defect mode. (d) Schematic drawing of the mechanism for dropping photons via coupling between a highly confined waveguide and localized cavity modes. 

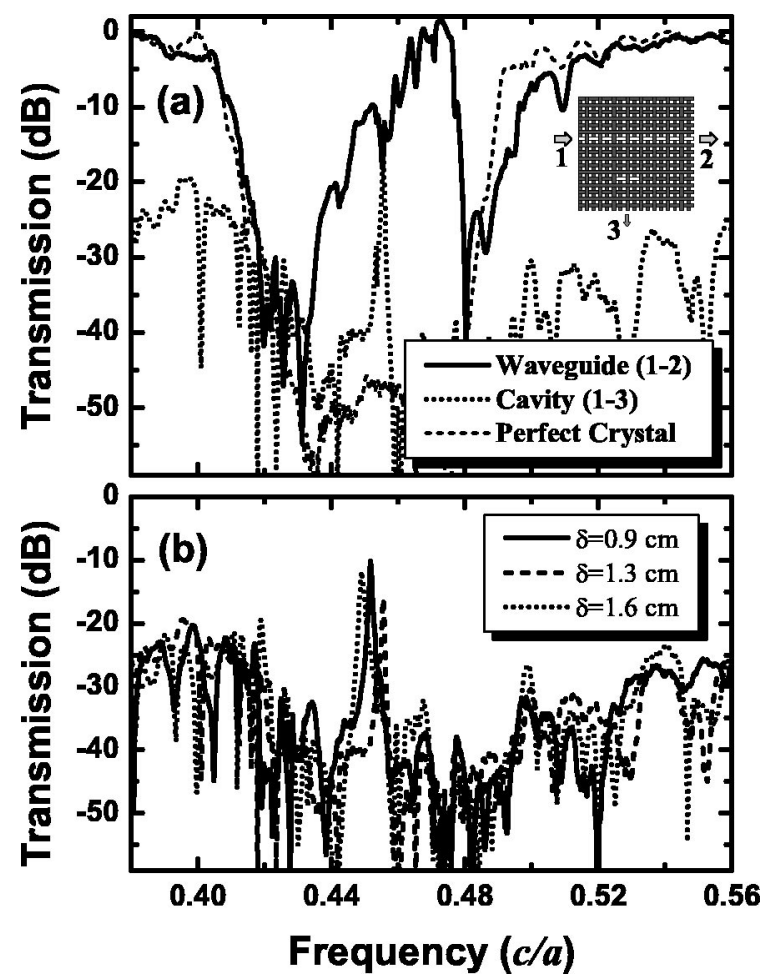

FIG. 2. (a) Measured transmission characteristics of the demultiplexing structure in Fig. 1(b). Photons with frequency $f=0.4555 \mathrm{c} / a$ are dropped from the waveguide mode. There is a corresponding drop in the transmission spectrum of the waveguide mode at the same resonance frequency. The transmission spectrum of the perfect crystal is also plotted for comparison. Inset: Schematic drawing of the demultiplexing geometry with parameters $\delta=1.3 \mathrm{~cm}$ and $\Delta=4 a$. (b) Measured transmission spectra corresponding to various values of $\delta$ for fixed $\Delta$. Tunability of the dropping frequencies can be achieved by varying $\delta$.

photonic band gap that extends from 10.6 to $12.8 \mathrm{GHz} .{ }^{24}$ The experimental setup consists of an HP $8510 \mathrm{C}$ network analyzer and microwave horn antennas to measure the transmission-amplitude spectra. The electric-field polarization vector of the incident EM field is always parallel to the stacking direction of the layers for all measurements.

In order to demonstrate the demultiplexing phenomena, we designed a structure that consists of a HCW and cavities [see Figs. 1(b) and 1(c)]. The highly localized defect modes, with quality factors $\left(Q=\omega_{0} / \Delta \omega\right)$ around 1000 , are generated either by removing some portion of the rod or by adding additional materials to the crystal. ${ }^{25-27}$ Due to coupling between the guided mode inside the waveguide and the localized cavity modes, the EM waves at the resonance frequencies of the cavities are dropped from the waveguide mode [See Fig. 1(d)].

The waveguide is constructed by removing a single rod from the 11th layer of the crystal which contains 5 unit cells along the stacking direction. Then, we construct an acceptorlike defect by cutting some portion of the rod which is at the same layer as the waveguide [Fig. 1(b)]. The defect volume, and therefore the cavity frequency, is varied by changing $\delta$. The distance between the defect and the waveguide is fixed at $\Delta=4 a$.

We first measure the transmission through the waveguide $(1 \rightarrow 2)$ (see the inset in Fig. 2). As shown in Fig. 2(a), the $\mathrm{HCW}$ has a guiding band (solid line) extending from $f$ $=0.437 \mathrm{c} / \mathrm{a}$ to $0.481 \mathrm{c} / \mathrm{a}$. The transmission spectrum exhibits

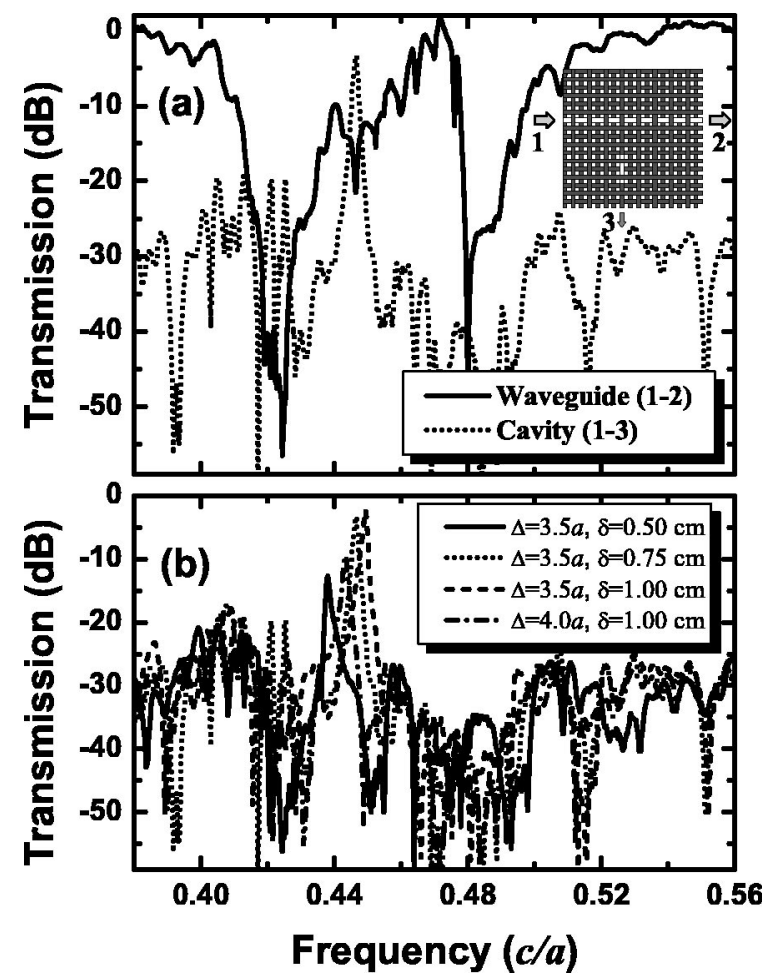

FIG. 3. (a) Measured transmission characteristics of the demultiplexing structure in Fig. 1(c). EM waves with frequency $f=0.4464 c / a$ are dropped from the HCW mode. There is a corresponding drop in the transmission spectrum of the waveguide mode at the same frequency. Inset: Schematic drawing of the demultiplexing geometry. (b) Measured transmission spectra corresponding to the various values of parameters $\Delta$ and $\delta$.

a dip at frequency $f=0.4555 \mathrm{c} / \mathrm{a}$. We then measure the transmission (dotted line) through the cavity $(1 \rightarrow 3)$. As shown in Fig. 2(a), a transmission peak (with a quality factor around 700) appears in the spectrum at the same frequency as the dip in the waveguide spectrum. For comparison, the transmission spectrum of a perfect crystal is also displayed in Fig. 2(a) which exhibits a stop band between $f=0.407$ and $0.490 c / a$.

Based on this observation, we conclude that the photons at the resonance frequency of the defect mode are first trapped in the cavity and then are emitted from the cavity. The quality factor of the dropping mode can be adjusted by changing the distance $\Delta$. Since the resonance frequency depends on the defect volume, we can tune the dropping frequency by increasing or decreasing the distance $\delta$. In order to demonstrate the tunability of our WDM structures, we measure the transmission spectra for three different values of $\delta$, $0.9,1.3$, and $1.6 \mathrm{~cm}$. As shown in Fig. 2(b), the dropping frequency can be tuned by changing the value of $\delta$.

The second demultiplexing structure is displayed in Fig. 1(c). In this configuration, the cavity is formed by cutting some portion of a rod at the upper layer (12th layer) of the $\mathrm{HCW}$ layer. The measured transmission characteristics are plotted in Fig. 3(a) for parameters $\Delta=3.5 a$ and $\delta$ $=0.75 \mathrm{~cm}$. The $\mathrm{EM}$ waves, with resonance frequency $f$ $=0.4464 c / a$, are filtered from the waveguide mode. We also observe a corresponding dip in the HCW spectrum at the same frequency. The tunability of the dropping mode is presented in Fig. 3(b). We measured the transmission spectra by varying parameters $\Delta$ and $\delta$. As shown in Fig. 3(b), the reso- 


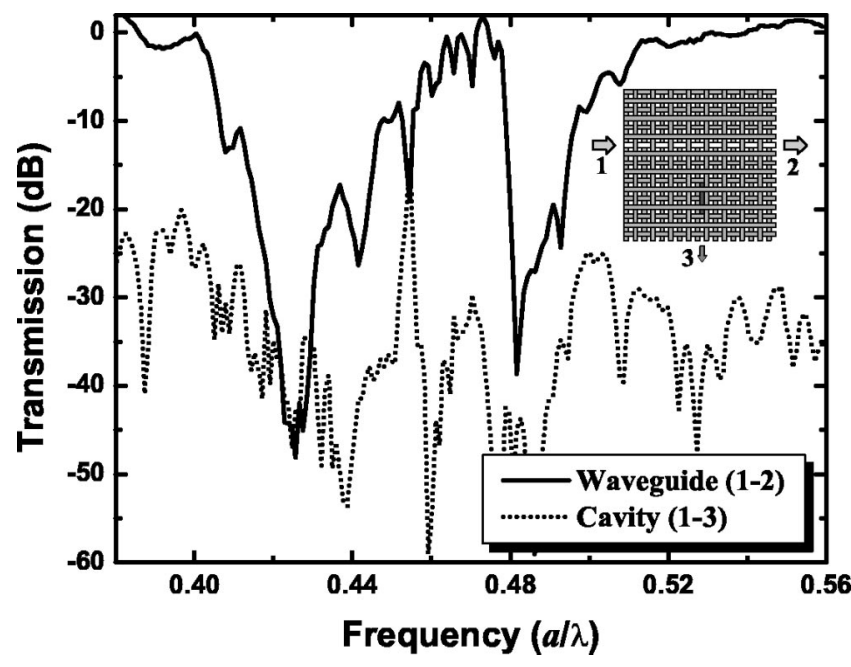

FIG. 4. Measured transmission characteristics of the donor-type demultiplexing structure. The electromagnetic wave at resonance frequency $f$ $=0.4545 \mathrm{c} / \mathrm{a}$ is dropped from the waveguide mode. Inset: Schematics of the demultiplexing geometry in which the cavity is formed by adding extra material (black rod).

nance frequency is shifted by changing parameters $\Delta$ and $\delta$.

We also investigate the demultiplexing phenomena by introducing donor-like defects into the crystal. An additional piece of rod of length $\delta=1.3 \mathrm{~cm}$ is placed at the 12th layer, at a distance $\Delta=4 a$ from the HCW (see the inset in Fig. 4). The measured transmission spectrum is displayed in Fig. 4. We observe dropping of a selective frequency similar to in the acceptor-like defect case. The EM waves at resonance frequency of $f=0.4545 \mathrm{c} / \mathrm{a}$ are filtered from the waveguide mode through the donor-like defect. The waveguide mode has a well-defined dip at the same frequency.

It is important to note that the quality factor of the dropping mode depends on the properties of the cavity and the coupling between the waveguide and the cavity modes. Moreover, by using the method reported by Fan and his coworkers, we can increase the transmission amplitude of the dropping mode ${ }^{9,28}$ by introducing accidental degeneracy into the system. For instance, by using two weakly interacting cavities, one can achieve complete dropping of the selected frequency from the waveguide mode.

In conclusion, we measured microwave transmission spectra corresponding to WDM structures in 3D photonic crystals. Photons with a certain wavelength are dropped from the waveguiding mode due to the coupling between the cavity and the highly confined waveguide. Our results can be used for designing filters and WDM components in future ultrasmall optical circuits.
This work was supported by NATO Grant No. SfP971970 and European Union Project EU-DALHM.

${ }^{1}$ For a recent review, see, for example, Photonic Crystal and Light Localization in the 21st Century, edited by C. M. Soukoulis (Kluwer, Dordrecht, 2001).

${ }^{2}$ S. Y. Lin, E. Chow, V. Hietala, P. R. Villeneuve, and J. D. Joannopoulos, Science 282, 274 (1998).

${ }^{3}$ M. Bayindir, B. Temelkuran, and E. Ozbay, Phys. Rev. B 61, R11855 (2000).

${ }^{4}$ M. Bayindir, E. Ozbay, B. Temelkuran, M. M. Sigalas, C. M. Soukoulis, R. Biswas, and K.-M. Ho, Phys. Rev. B 63, 081107 (2001).

${ }^{5}$ T. F. Krauss and R. M. D. L. Rue, Appl. Phys. Lett. 68, 1613 (1996).

${ }^{6}$ M. Bayindir, B. Temelkuran, and E. Ozbay, Appl. Phys. Lett. 77, 3902 (2000)

${ }^{7}$ Y. Sugimoto, N. Ikeda, N. Carlsson, K. Asakawa, N. Kawai, and K. Inoue, Opt. Lett. 27, 388 (2002).

${ }^{8}$ O. Painter, R. K. Lee, A. Scherer, A. Yariv, J. D. O’Brien, P. D. Dapkus, and I. Kim, Science 284, 1819 (1999).

${ }^{9}$ S. Fan, P. R. Villeneuve, J. D. Joannopoulos, and H. A. Haus, Phys. Rev. Lett. 80, 960 (1998)

${ }^{10}$ H. Kosaka, T. Kawashima, A. Tomita, M. Notomi, T. Tamamura, T. Sato, and S. Kawakami, Appl. Phys. Lett. 74, 1370 (1999).

${ }^{11}$ S. Noda, A. Chutinan, and M. Imada, Nature (London) 407, 608 (2000).

${ }^{12}$ B. E. Nelson, M. Gerken, D. A. B. Miller, R. Piestun, C.-C. Lin, and J. S. Harris, Opt. Lett. 25, 1502 (2000).

${ }^{13}$ S. S. Oh, C.-S. Kee, J.-E. Kim, H. Y. Park, T. I. Kim, I. Park, and H. Lim, Appl. Phys. Lett. 76, 2301 (2000).

${ }^{14}$ M. Koshiba, J. Lightwave Technol. 19, 1970 (2001).

${ }^{15}$ A. Sharkawy, S. Shi, and D. W. Prather, Appl. Opt. 40, 2247 (2001).

${ }^{16}$ C. Jin, S. Han, X. Meng, B. Cheng, and D. Zhang, J. Appl. Phys. 91, 4771 (2002).

${ }^{17}$ S. G. Johnson, P. R. Villeneuve, S. Fan, and J. D. Joannopoulos, Phys. Rev. B 62, 8212 (2000).

${ }^{18}$ J. G. Fleming and S.-Y. Lin, Opt. Lett. 24, 49 (1999).

${ }^{19}$ S. Noda, K. Tomoda, N. Yamamoto, and A. Chutinan, Science 289, 604 (2000).

${ }^{20}$ S. Y. Lin, J. G. Fleming, D. L. Hetherington, B. K. Smith, R. Biswas, K. M. Ho, M. M. Sigalas, W. Zubrzycki, S. R. Kurtz, and J. Bur, Nature (London) 394, 251 (1998).

${ }^{21}$ M. M. Sigalas, R. Biswas, K.-M. Ho, C. M. Soukoulis, D. Turner, B. Vasiliu, S. C. Kothari, and S. Lin, Microwave Opt. Technol. Lett. 23, 56 (1999).

${ }^{22}$ A. Chutinan and S. Noda, Appl. Phys. Lett. 75, 3739 (1999).

${ }^{23}$ K. M. Ho, C. T. Chan, C. M. Soukoulis, R. Biswas, and M. M. Sigalas, Solid State Commun. 89, 413 (1994).

${ }^{24}$ E. Ozbay, J. Opt. Soc. Am. B 13, 1945 (1996)

${ }^{25}$ One calls these modes acceptor- or donor-like photonic modes which are reminiscent of the acceptor and donor states in a semiconductor (see Refs. 26 and 27).

${ }^{26}$ E. Yablonovitch, T. J. Gmitter, R. D. Meade, A. M. Rappe, K. D. Brommer, and J. D. Joannopoulos, Phys. Rev. Lett. 67, 3380 (1991).

${ }^{27}$ E. Ozbay, G. Tuttle, M. M. Sigalas, C. M. Soukoulis, and K. M. Ho, Phys. Rev. B 51, 13961 (1995).

${ }^{28}$ S. Fan, P. R. Villeneuve, J. D. Joannopoulos, M. J. Khan, C. Manolatou, and H. A. Haus, Phys. Rev. B 59, 15882 (1999). 